\title{
ARQUITECTURA COMO ESPACIO PARA LA UTOPÍA, EL CASO DEL INSTITUTO DE INVESTIGACIONES CIENTÍFICAS EN LA PLATA, ARGENTINA (1968-1973)
}

\author{
ARCHITECTURE AS A SPACE \\ FOR UTOPIA: THE CASE OF THE INSTITUTO \\ DE INVESTIGACIONES CIENTÍFICAS, \\ LA PLATA, ARGENTINA (1968-1973)
}

\author{
CECILIA PARERA \\ Universidad Nacional del Litoral, Argentina
}

La sede del Instituto de Investigaciones Científicas de la Universidad Nacional de La Plata, en Argentina, remite a dos utopías, en tanto horizontes a alcanzar. En el plano político, expresa el impulso a la investigación como parte del fortalecimiento económico del modelo estatal desarrollista, mientras que en lo arquitectónico constituye una manifestación temprana de la "arquitectura de sistemas" dejando en evidencia una acabada confianza en la tecnología. El artículo analiza una obra poco atendida, ilustrativa de un período disciplinar fructífero en ideas optimistas sobre el futuro, a la vez que testimonio del quiebre respecto de la arquitectura moderna de entreguerra.

utopía tecnológica, arquitectura de sistemas, Argentina, universidad, Estado desarrollista
Recibido: 30 de mayo del 2019

Aprobado: 5 de agosto del 2019

doi: 10.26439/limaq2020.n006.4814

The Instituto de Investigaciones Científicas of the Universidad Nacional de La Plata in Argentina refers to two utopias according to the horizons to be reached. At the political level, it expresses the boost given to research, as part of the proposals for economic strengthening of the government's developmental model. At the architectural level, it is one of the earliest manifestations of "system architecture," evidencing confidence in technology. This article analyzes a work often ignored: an illustrative work of a productive disciplinary period with regard to optimistic ideas about future development and witness of the turning point of interwar modern architecture.

technological utopia, system architecture, Argentina, university, developmental state 


\section{EL DESARROLLO COMO UTOPÍA DEL ESTADO EN LATINOAMÉRICA}

En el contexto económico-productivo de la segunda posguerra, las teorías económicas clásicas fueron cuestionadas ante el reconocimiento del marcado deterioro en los términos de intercambio, que actuaba en perjuicio de aquellas economías primario-exportadoras. En este período, en Latinoamérica se manifestaron diversas interpretaciones, particularmente en el marco de la Comisión Económica para América Latina (CEPAL), las que identificaban que las características sociopolíticas propias de la región tendían a obstaculizar su desarrollo económico. Estas respuestas teóricas se transformaron en los pilares conceptuales de un nuevo modelo estatal, el "desarrollismo", que se fundó a través del impulso de las políticas de industrialización por sustitución de importaciones a fin de reducir la dependencia del mercado interno respecto de bienes con alto valor agregado provenientes del exterior. Según este posicionamiento, la progresiva asimilación de la técnica moderna mejoraría el rendimiento y la racionalización de la estructura productiva de los países "en desarrollo" (Altamirano, 1998). De esta manera, el Estado asumía el rol de controlador de las políticas de inversión, definiendo las áreas económicas que debían ser fomentadas.

En la década de 1950 diversos gobiernos latinoamericanos asumieron estos lineamientos impulsando sectores clave de las industrias básicas, como la petrolera, la química, la metalúrgica y la maquinaria creando una serie de empresas estratégicas para lograr el equilibrio del desarrollo nacional. Las gestiones de Adolfo Ruiz Cortines en México (período 1952-1958), Carlos Ibáñez del Campo en Chile (1952-1958), Juscelino Kubitschek en Brasil (1956-1961) y Arturo Frondizi en Argentina (1958-1962) son testimonio del referido modelo.

Para alcanzar los objetivos planteados, la promoción de la investigación y la formación de científicos se constituyeron en estrategias centrales (Yocelevsky, 2015). En este contexto, es posible entender la proliferación de instituciones estatales dedicadas a la investigación científica, como el Instituto Nacional de Tecnología Industrial en Argentina (creado en 1956), el Consejo de Desarrollo del Plan de Metas en Brasil (1956) y la Comisión Nacional de Investigaciones Científicas y Tecnología en Chile (1966), los que se centraron en diseñar soluciones para resolver, respectivamente, la debilidad del sector industrial, la dependencia de la economía y la falta de organización del sistema científico y tecnológico local.

A su vez, la apuesta pública se cristalizó en una serie de medidas tendientes a impulsar la formación universitaria de técnicos capaces de brindar respuestas adecuadas a las principales demandas definidas por la agenda estatal, afrontando en paralelo el marcado crecimiento de la matrícula universitaria y la demanda por espacios físicos específicos para la optimización de las investigaciones científicas y los desarrollos tecnológicos. La creación de nuevas instituciones de formación superior, como la Universidad de Lima en Perú (1962) y la Universidad Nacional de Rosario en Argentina (1968), así como 
los proyectos para nuevas edificaciones en las Universidades de Concepción en Chile (1957) y de Brasilia en Brasil (1963) fueron algunos de los tantos testimonios de estas políticas.

\section{LAS UNIVERSIDADES, CENTRO DEL DEBATE ARQUITECTÓNICO}

En Argentina la década de 1960 fue clave para las instituciones de formación superior, las que se encontraban sumidas en un profundo debate en torno a la revisión de las estrategias educativas compartimentadas por especialidades y la necesidad de apertura al medio social y a la estructura productiva del país. En menos de una década la creación de universidades privadas - no permitidas hasta 1955, como la Universidad Católica de Santa Fe y la Universidad Argentina de la Empresa- y la ampliación de la cobertura geográfica - habilitando nuevas universidades en regiones desprovistas, entre ellas Entre Ríos, Rosario y San Juan-, permitieron duplicar la oferta académica (Buchbinder, 2005).

Este proceso fue seguido por espasmódicas estrategias de concreción de ámbitos físicos de las reparticiones técnicas de cada institución, signadas por los cambios en relación con las políticas universitarias definidas en el período, particularmente restrictivas en el marco de los trágicos golpes militares de 1962 y $1966^{1}$. Los proyectos para ciudades universitarias, como las de Buenos Aires, en 1959, y de Tucumán, en 1964, ponían en evidencia cuestiones clave para la arquitectura como la vinculación social con la ciudad existente o su capacidad de impulsar el crecimiento urbano (Gentile, 2004) ${ }^{2}$. Por otro lado, las propuestas para la construcción de nuevas sedes como la Facultad de Ciencias Políticas y Sociales en la Universidad Nacional de Cuyo (arquitecto Juan Brugiavini, 1966) y la Facultad de Ciencias Exactas, Físicas y Naturales en la Universidad Nacional de Córdoba (arquitectos Oscar Acuña, Mario Damond y Arquímedes Federico, 1972), permitieron reconocer discusiones centrales para la disciplina, en particular con relación a la escala, la organización programática y la incorporación de nuevas tecnologías (Malecky, 2014).

1 Particularmente en el ámbito universitario el golpe de Estado encabezado en 1966 por el general Juan Carlos Onganía, que derrocó el gobierno constitucional del presidente Arturo Illía (1963-1966), constituyó un cisma significativo. Una de sus primeras medidas fue la intervención de las universidades y la anulación de su régimen de autonomía. Es así que la Noche de los Bastones Largos, como fue denominado el violento desalojo de numerosas facultades del país en julio de 1966, fue el principal acontecimiento de una etapa compleja para la educación superior.

2 Más allá de la multiplicación de proyectos relacionados con este programa funcional, el interés sobre la temática por estos años en el ámbito de la arquitectura queda de manifiesto en la organización de jornadas y seminarios sobre ciudades universitarias en 1970 y 1971, así como en la publicación de ediciones especiales en revistas disciplinares, entre ellas la revista Summa con su número "Ámbito físico de las universidades argentinas" de 1974. 
당

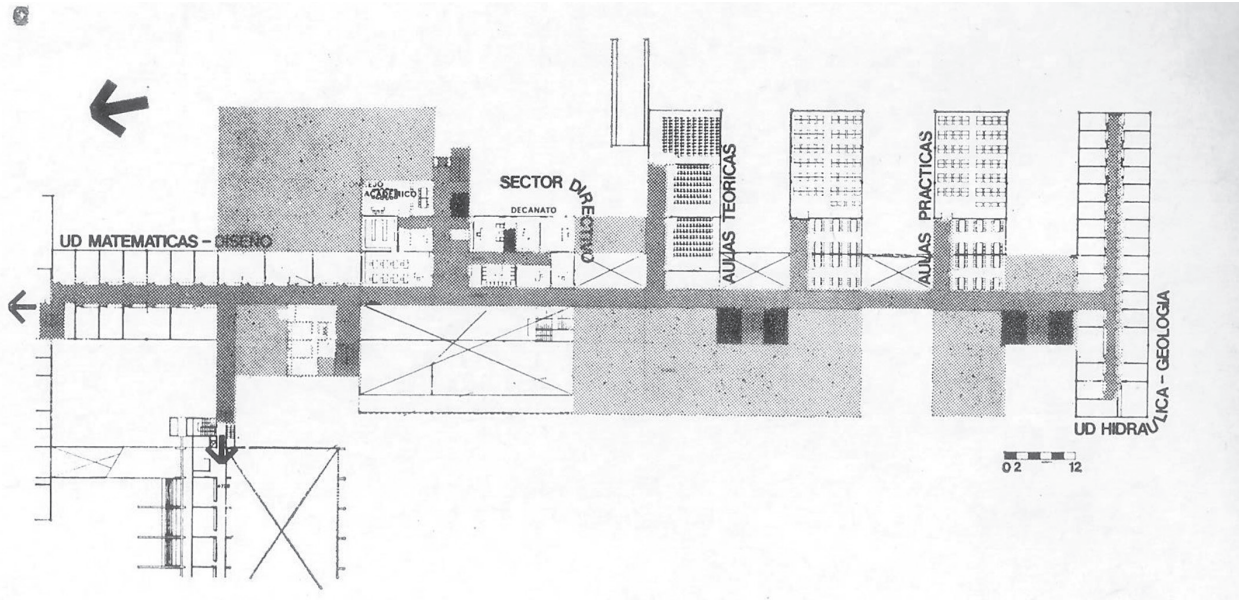

Figura 1

Planta nivel $+6,70 \mathrm{~m}$, Facultad de Ciencias

Exactas, Físicas

y Naturales,

Universidad

Nacional de

Córdoba

Fuente: Summa,

1974 , p. 38
En el ámbito internacional, los proyectos para los campus de la Universidad Libre de Berlín en Alemania (arquitectos Georges Candilis, Alexis Josic y Sharach Woods, 1963) y de la Universidad de Hull en Inglaterra (arquitecto Leslie Martin, 1962) ponían de manifiesto una nueva estrategia de abordaje que resolvía las nuevas demandas - particularmente las obras de alta complejidad - con "una gran estructura en la que tienen cabida todas las funciones... La tecnología actual la ha hecho posible", retomando los términos con los que el arquitecto japonés Fumihiko Maki definía a las megaestructuras en 1964 (Banham, 2001, p. 8). En línea con el pensamiento estructuralista característico del período (el cual ponderaba la relación entre los elementos por sobre los objetos en sí mismos), en el campo de la arquitectura se incorporó la noción

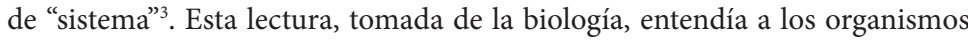
como conjuntos con propiedades específicas que no podían ser reducidos a las partes de sus componentes y en los que las relaciones entre ellos asumían un rol preponderante.

El nuevo paradigma proyectual, que tuvo su principal desarrollo en Inglaterra de la mano de arquitectos como Sharach Woods y Cedric Price atendía a "clarificar el proceso de diseño, descomponer las partes del programa, poder visualizar científicamente sus propiedades para luego poder re-ensamblarlas con certeza" (Aliata, 2014, p. 15). Resulta relevante señalar que en las décadas de 1950 y 1960 en Argentina la influencia de esta estrategia alcanzó gran difusión de la mano de revistas europeas (mayormente inglesas, como Architectural Review y Architectural Design) así como locales (Summa, Nuestra

3 En la década de 1950 la teoría general de los sistemas había alcanzado las más diversas aplicaciones intentando dar una respuesta a la creciente complejidad que afectaba al mundo industrializado. En el campo de la arquitectura, las estrategias de reconversión de la industria bélica aplicada a la renovación edilicia, la presión por rápidas respuestas a la estructura productiva y la amplia disponibilidad de nuevas tecnologías y sistemas constructivos, entre otros factores, impulsaron el mencionado cambio de paradigma proyectual (Baudizzone, 1975b). 
Arquitectura, Trama). En particular, los mencionados proyectos dejaban en evidencia un quiebre respecto de la lógica racionalista, consolidada en la entreguerra, que buscaba organizar las actividades de manera funcional al extremo y en la que la forma celebraba la precisión técnica. Un cambio que fue sustancial para los profesionales intervinientes en el campo de los proyectos por estos años: "Pertenecemos a una generación de arquitectos formada bajo el entusiasta manifiesto de la arquitectura moderna, pero también bajo la desconfianza de la aceptación acrítica de su doctrina, iniciada en la década de 1960" (Baudizzone, M., Erbin, J., Lestard, J., Varas, A., 1980, p. 15).

El proyecto para la Facultad de Ciencias Exactas de la Universidad Nacional de La Plata (UNLP) constituye un caso paradigmático en torno a los mencionados debates. En 1967 la universidad había creado la Dirección de Obras y Planeamiento para evaluar alternativas de expansión edilicia, concentrándose en la reorganización integral de su campus en los terrenos del Bosque (Gandolfi y Gentile, 1997). Como parte del plan de obras se contrató al estudio porteño integrado por los arquitectos Miguel Baudizzone, Jorge Erbin, Jorge Lestard, Eithel Traine, Alberto Varas y Juan Baliester Peña (BELTVB), joven equipo que ya había incursionado exitosamente en numerosos concursos y se encontraba construyendo la Facultad Regional de la Universidad Tecnológica Nacional en Avellaneda, provincia de Buenos Aires.

Figura 2.

Axonometría del proyecto para la Facultad de Ciencias

Exactas, UNLP

Fuente: Summa, 1971, p. 23

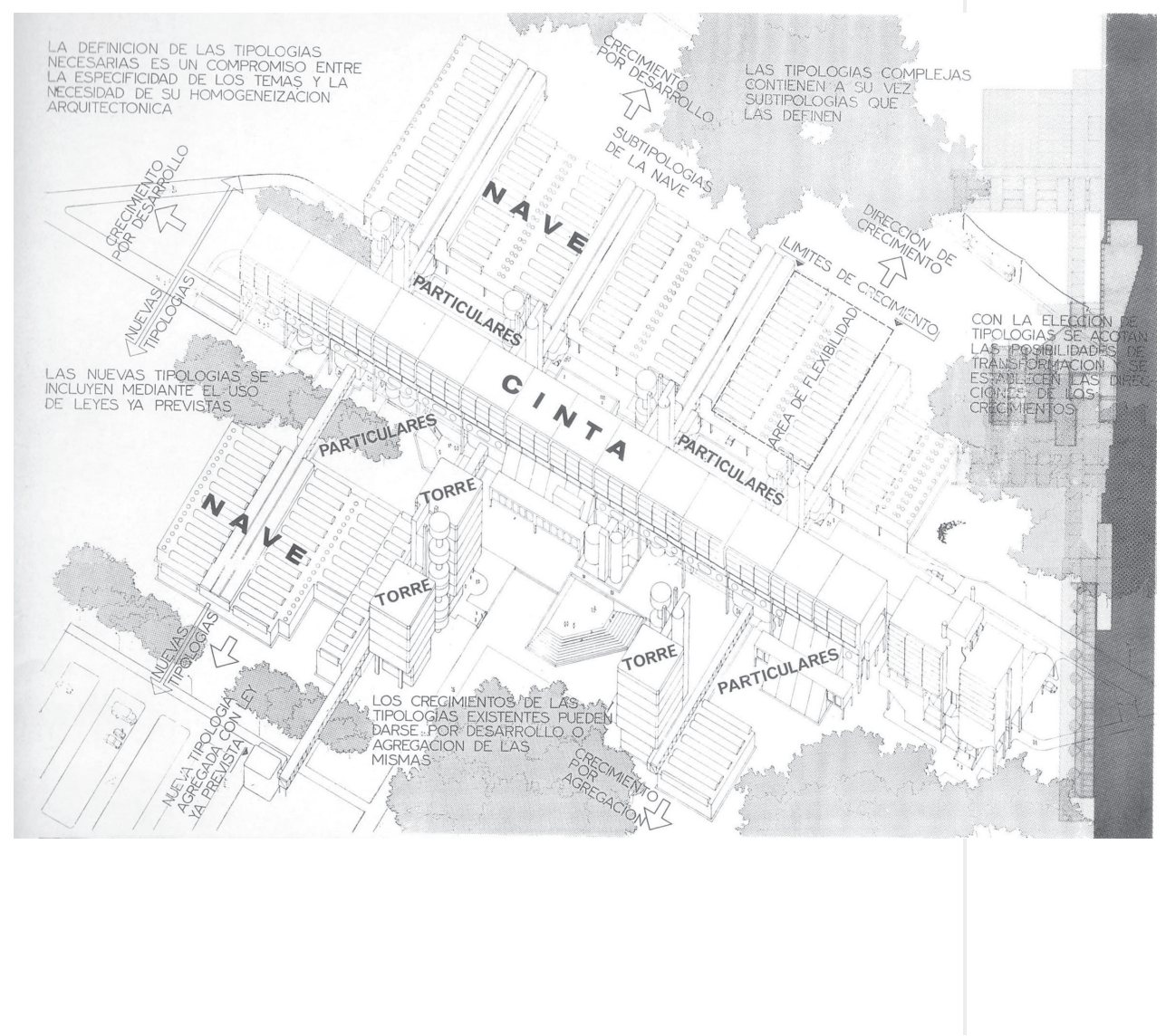


La propuesta fue estructurada a partir de la generación de una trama de circulaciones, eje cívico al cual se integraban los distintos componentes del programa de necesidades, como aulas teóricas generales, laboratorios de docencia masiva, biblioteca y gabinetes. Previendo posibles variaciones cuantitativas y cualitativas en los requerimientos físicos para el desarrollo de la enseñanza y la investigación, cada pieza podía ser transformada o reemplazada. Por su parte, el eje principal podía extenderse en horizontal hacia el noroeste del predio, a la manera de propuestas contemporáneas como Plugin-city y City-Interchange del equipo británico Archigram.

Sobre la base de la determinación de las condiciones óptimas para el desarrollo de las actividades y de los niveles deseables de complejidad, eficiencia o interacción del conjunto se pueden determinar, por agrupamiento o segregación, tipologías ambientales que, en definitiva, componen el edificio como partes elementales. (Erbin y Varas, 1974, p. 21)

Figura 3

Planta tipo del área de

laboratorios y corte longitudinal

del Instituto de

Investigaciones

Científicas,

UNLP

Fuente: Summa,

1974 , p. 77

\section{UNA UTOPÍA CONSTRUIDA: EL INSTITUTO DE INVESTIGACIONES CIENTÍFICAS UNLP}

En el extremo sureste del conjunto se previó la ubicación del Instituto de Investigaciones Científicas ${ }^{4}$, único edificio del ambicioso proyecto que sería concretado. Esta pieza fue concebida con las mismas ideas que guiaron el proyecto general de la facultad, estructurando el programa a partir de un tronco vertical de circulaciones y sanitarios. A este elemento vertebrador se anexaron los volúmenes propios de cada tipología espacial, "fragmentos convertidos en unidad de diseño" (Waisman, 1995, p. 101). Las áreas de mayor flujo de asistentes fueron dispuestas en zonas próximas al ingreso, como los dos auditorios en los pisos inferiores, mientras que los laboratorios, de acceso más restringido, fueron elevados. Por su parte, una amplia superficie de depósitos en planta baja fue distanciada de la torre y dispuesta para un fácil acceso de los proveedores.
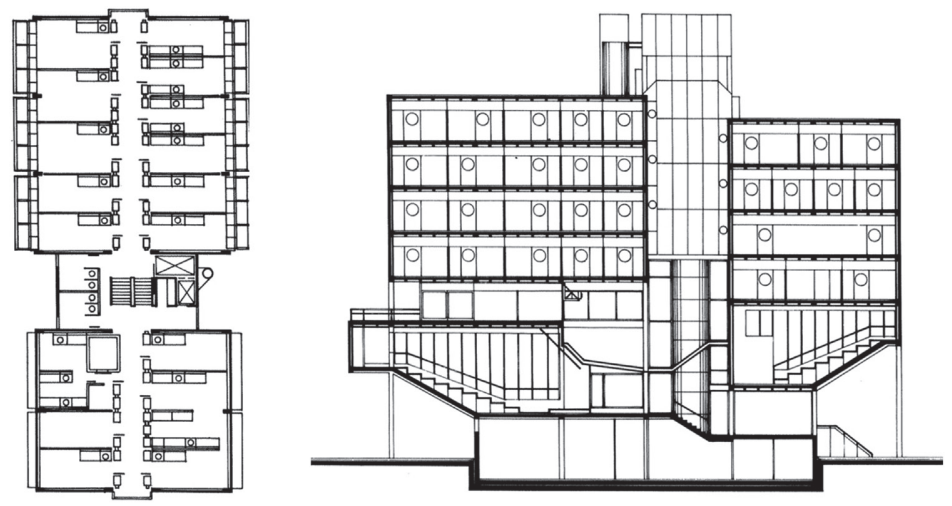

4 Esta dependencia en la actualidad se denomina Instituto de Investigaciones Fisicoquímicas Teóricas y Aplicadas (INIFTA), dependiente de la Universidad Nacional de La Plata y del Consejo Nacional de Investigaciones Científicas y Tecnológicas (Conicet). 
Siguiendo la mencionada lógica, los laboratorios fueron agrupados según la actividad que se desarrollaba en su interior. El bloque más alto nuclea los espacios para el trabajo cotidiano de los investigadores, por lo que la superficie vidriada es mayor, y el equipamiento y los paneles divisorios son desmontables. El otro bloque está destinado a alojar equipos pesados para el desarrollo de experimentos. Allí la permanencia de científicos es acotada por lo que el ingreso de iluminación y ventilación naturales es controlado con delgadas ventanas horizontales protegidas de la radiación solar con viseras. El vínculo entre ambos cuerpos se establece a partir de tramos de escaleras de medio nivel, lo que amplía las opciones de utilización de los equipos. Las instalaciones especiales fueron distribuidas en cielorrasos técnicos que llegaban a cada piso a partir de conductos expuestos de chapa pintada, dando respuesta al reclamo de Reyner Banham por "superar el ocultamiento de los servicios" (Banham, 1969, p. 238). Por estos años, las ideas de este crítico inglés habían alcanzado gran influencia en el debate disciplinar en Argentina, particularmente tras su visita al país en 1968 para dictar una serie de conferencias tituladas "El control ambiental en la arquitectura moderna”. El material presentado en sus disertaciones constituyó el insumo principal para el libro que publicaría un año después: La arquitectura del entorno bien climatizado (Parera, 2017).

Figura 4.

Exterior del Instituto de Investigaciones Científicas, UNLP

Archivo fotográfico de la autora

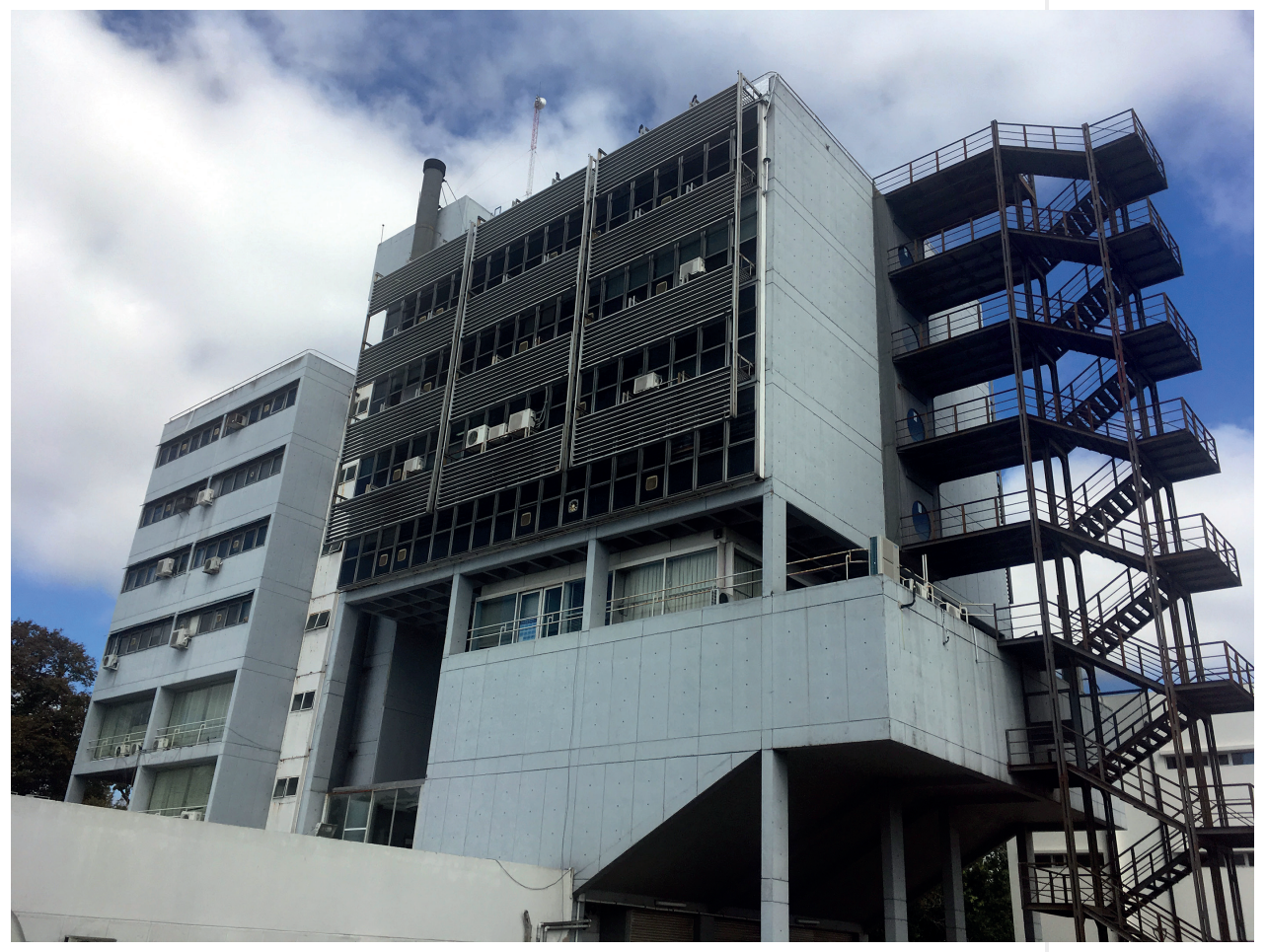


Figura 5

Área de acceso al auditorio mayor del Instituto de Investigaciones Científicas

de la UNLP

Archivo fotográfico de la autora

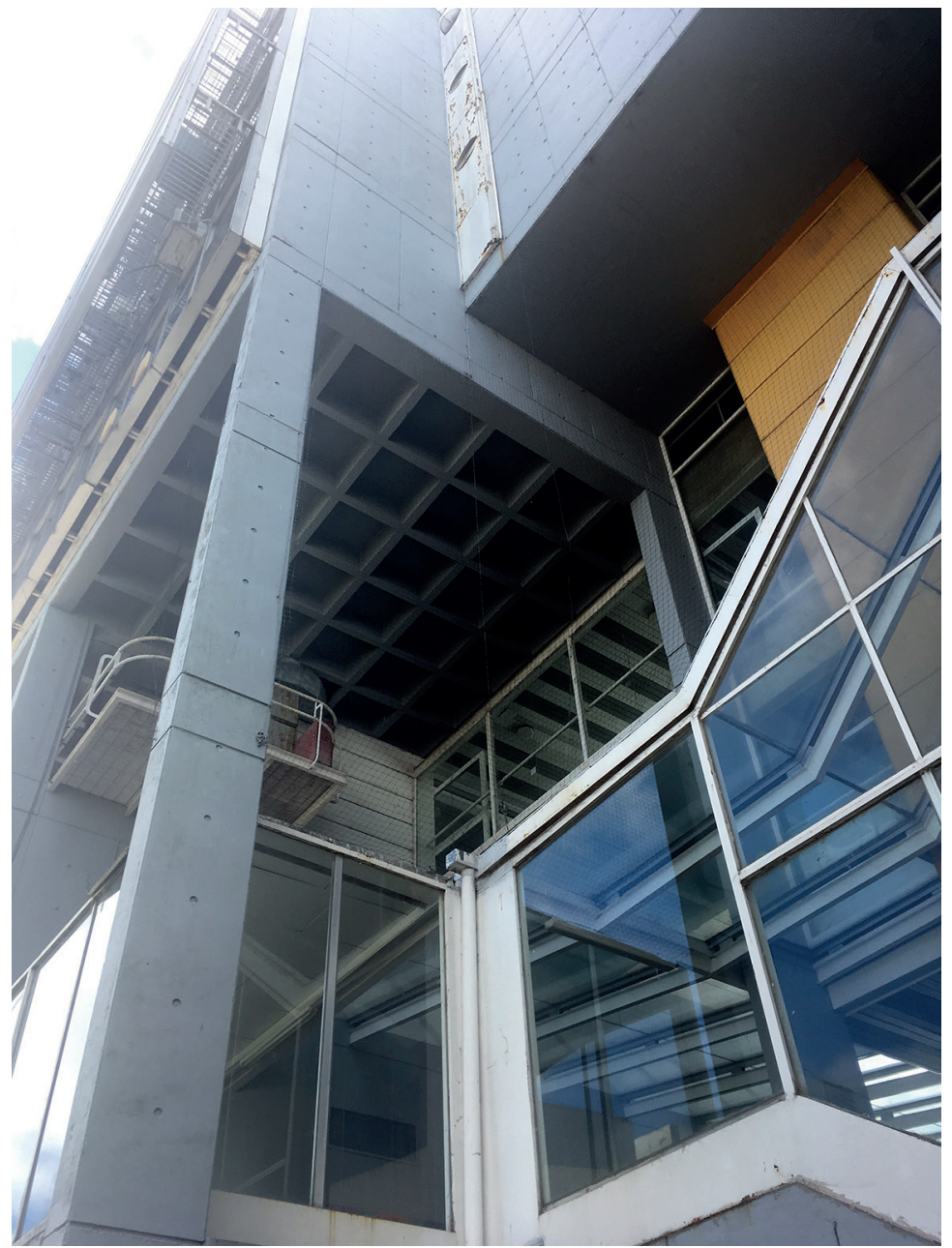

En términos formales, cada componente del programa se expresa de manera autónoma, sin necesidad de una composición unificadora. Es por ello que los auditorios se reconocen tanto por las pendientes de las gradas que definen sus caras inferiores como por sus laterales ciegos. Ingresos y vestíbulos, por su parte, se constituyen como cajas vidriadas, abriendo visuales al entorno y exhibiendo el dinamismo interior. Los materiales contribuyen a esta identificación; mientras paneles premoldeados de hormigón conforman casi la totalidad del cerramiento de los espacios que alojan a los equipos, una trama de parasoles de aluminio protege la superficie vidriada de los laboratorios de los investigadores.

En esta línea, la terminación de los paneles que cubren la torre de servicios fue resuelta con diferente trama de ensamblaje y tonalidad a fin de destacarla del resto de los componentes de hormigón. El área de depósito y talleres está 
revestida con una envolvente metálica removible de listones y bastidores que acentúa su naturaleza productiva e incorpora luz natural cenital al través de bóvedas corridas de poliéster moldeado con fibra de vidrio. Terrazas y balcones, cuales puestos fabriles de control, se asoman con sus barandas de caño pintado. El estudio BELTVB incorporó numerosas innovaciones buscando sistematizar las diferentes etapas de la obra y racionalizar el uso de materiales, cuestión que por estos años era un valor destacado por la mayoría de los profesores de los talleres de proyecto en la Facultad de Arquitectura y Urbanismo de la Universidad de Buenos Aires, institución en la cual los miembros del equipo habían recibido su título profesional (Molina y Vedia, 2004). De esta manera, dejaban en claro su confianza en el proceso de industrialización en el que se encontraba inmerso el país en general y el sector de la construcción en particular: "La posibilidad de producir en una región con un desarrollo industrial medio nos ha dado posibilidades de transitar soluciones no tradicionales, aprovechando sistemas existentes o adecuando tecnologías de otras industrias extrañas a la construcción” (Baudizzone, Díaz, Erbin, Lestard, Varas, 1975a, p. 34).

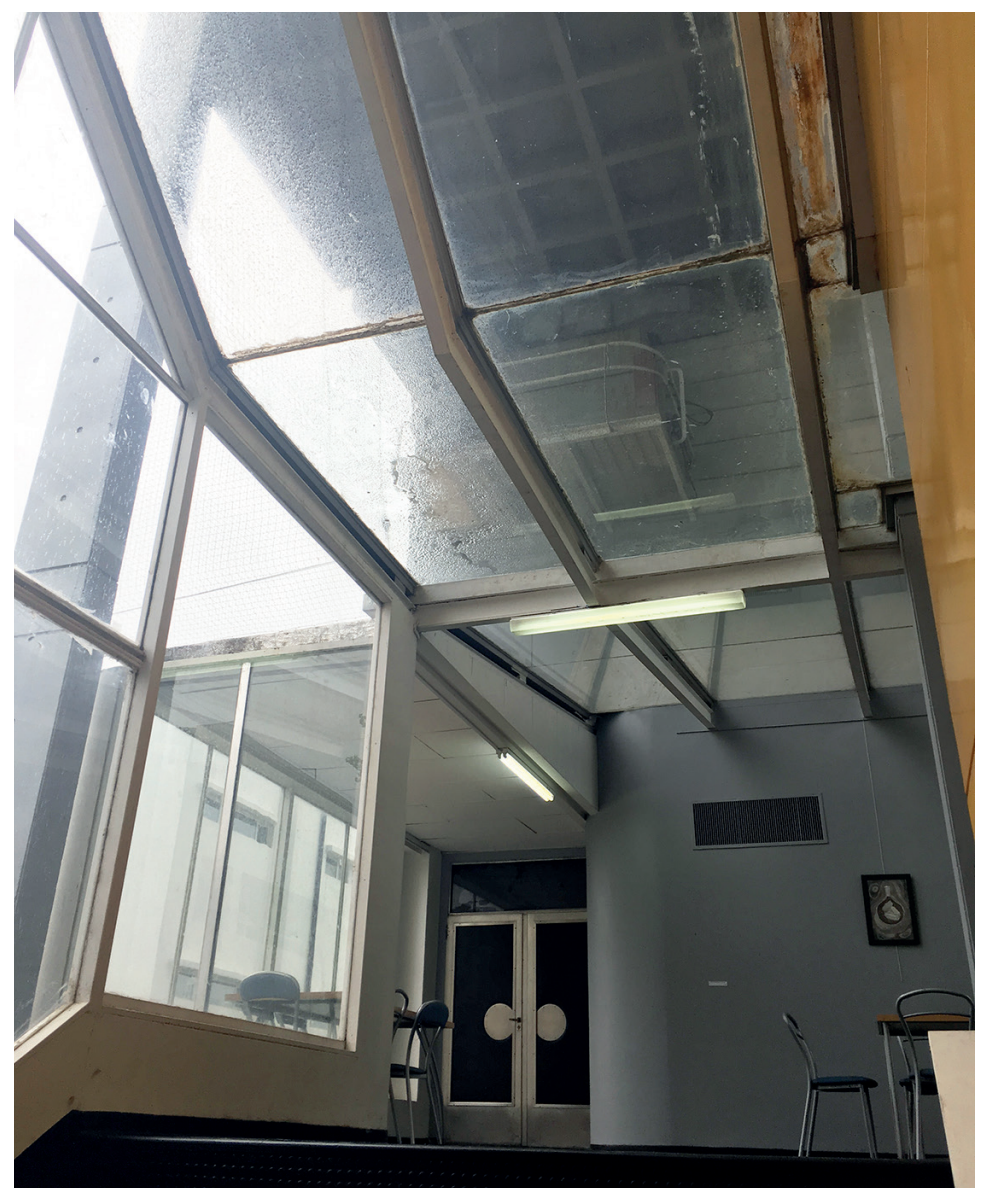

Figura 6.

Área de ingreso al auditorio mayor desde el vestíbulo, Instituto de Investigaciones Científicas de la UNLP 
Las alusiones industriales, la insinuación de indeterminación y la imagen fuertemente tecnológica de la obra dejan de manifiesto la ruptura con el código formal de "la primera era de la máquina" (Banham, 1960) ${ }^{5}$. A su vez, permiten identificar una afinidad estética con el pop art norteamericano, el independent group británico y el situationnisme francés, movimientos afines a los mass media, a la cultura del espectáculo y a la imaginería en torno a la vida cotidiana, ampliamente difundidos en Argentina por estos años (Plotkin, 2015).

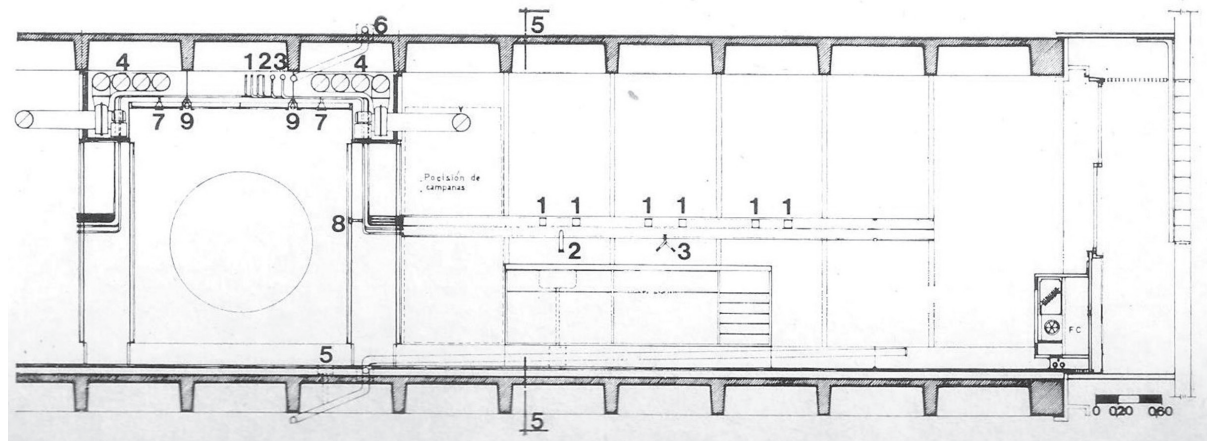

Figura 7

Detalle en corte de uno de los laboratorios para investigadores del Instituto de Investigaciones

Científicas,

UNLP

Fuente:

Summa, 1974

p. 77
Al observar la volumetría en su conjunto, la relación con la Facultad de Ingeniería de la Universidad de Leicester en Inglaterra resulta insoslayable. Esta obra, proyectada en 1959 por los arquitectos británicos James Stirling y James Gowan, se compone de una variedad de volúmenes independientes. Sobre la utilización de esta estrategia del proyecto reflexionaba Marina Waisman (1995): "Stirling compuso magistralmente sus obras con fragmentos coherentes en sí mismos, aligerados, eso sí, por un toque irónico o quizás simplemente festivo" (p. 101). El conjunto, con sus contornos acusados y alusiones industriales expresamente expuestas, se constituyó en una de las obras inglesas más difundidas del período. A su vez, indudablemente, resultó un referente adecuado para el estudio BELTVB, cuyos integrantes era confesos admiradores de la obra contemporánea de James Stirling (Baudizzone, entrevista personal, 2018). 


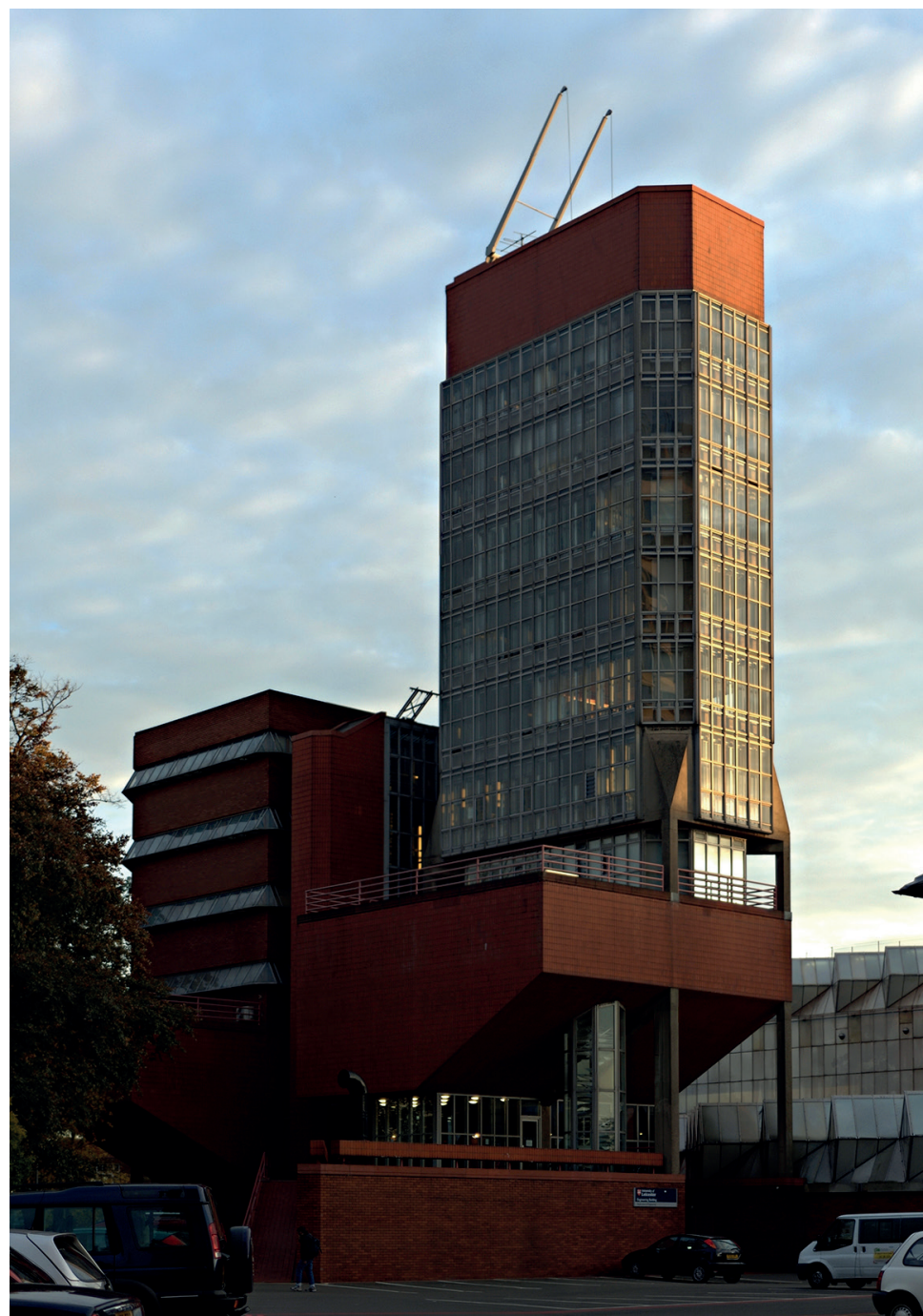

REFLEXIONES FINALES: LA TECNOLOGÍA COMO UTOPÍA PARA LA ARQUITECTURA

El edificio del Instituto de Investigaciones Científicas, obra destacada de la política estatal desarrollista, constituye un claro referente del quiebre respecto del "auge ideológico del funcionalismo ingenuo" (Baudizzone et al., 1980, p. 15). Esta obra se presenta como un temprano exponente de la "arquitectura de sistemas" en Argentina, estrategia que entre fines de la década de 1960 y principios de la siguiente dominaría los proyectos de arquitectura de alta complejidad en el país,
Figura 8.

Exterior de la

Facultad de

Ingeniería de

la Universidad

de Leicester en

Inglaterra

Fuente: Rickert, 2006, p. 73 
Figura 9

Área de ingreso del hospital San Vicente de Paul en Orán, provincia de Salta

Fuente: Rubén Cabrera, 2011 como es el caso del hospital San Vicente de Paul en Orán, provincia de Salta (arquitectos Juan Manuel Llauró y José Antonio Urgell, 1969-1977) y del Centro Cívico de la Provincia de San Juan (arquitectos Antonio Antonini, Juan Manuel Llauró, Gerardo Schön, José Antonio Urgell y Eduardo Zemborain, 1971-2009). Esta arquitectura, innegablemente, buscaba presentarse como el resultado de la firme creencia de sus autores en el progreso en manos de la técnica, a la que consideraban neutral y carente de fines retóricos (Silvestri, 2014).

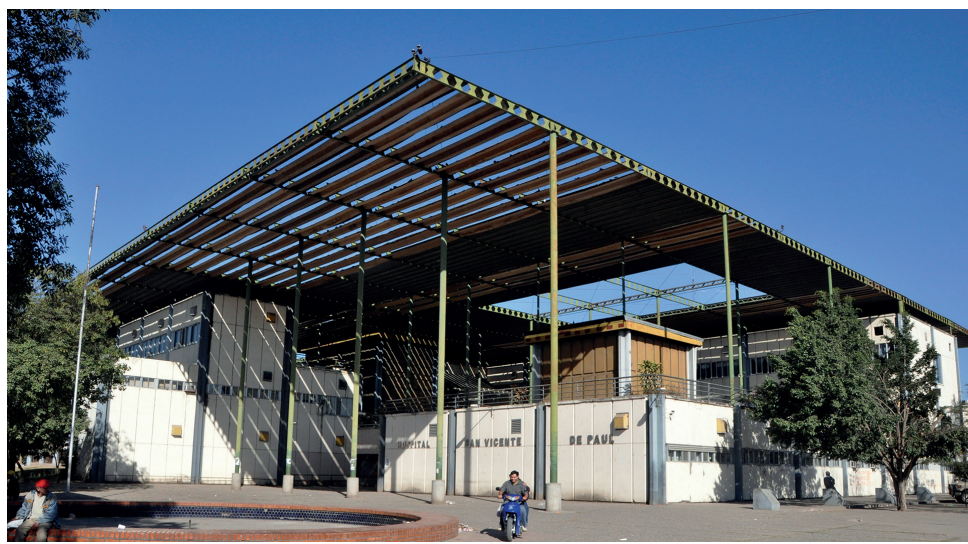

La preocupación por los aspectos tecnológicos de la obra del estudio BELTVB dista de implicar una actitud de distanciamiento respecto de otros valores como los formales, sociales o económicos. Como señala Felicity Scott en Architecture or Techno-utopia: Politics after modernism al estudiar una serie de obras de arquitectura de las décadas de 1960 y 1970 no es posible identificar en estas prácticas una aceptación ciega de la tecnología, como tampoco un rechazo rotundo a sus posibilidades (Scott, 2007). Más bien, todas ellas expresaban las contradicciones y dualidades propias de un período que quería mostrarse como superador de los postulados modernos que ya habían sido puestos en crisis con la revisión historiográfica del Movimiento moderno iniciada a finales de la década de 1940 (Tournikiotis, 2001). En el caso analizado en el presente artículo, como definieron sus arquitectos a cargo, se trataba de una actitud crítica en que la incorporación de lógicas provenientes de la producción industrial era asumida positivamente como elemento esencial de la práctica arquitectónica contemporánea:

El conocimiento, la investigación y la puesta en práctica de leyes generales de diseño es uno de los temas destinados a convertir a la arquitectura en una disciplina basada en óptimas respuestas a los usos, a la altura del actual desarrollo científico y técnico y con posibilidades de jugar un papel en la creación del ambiente físico. (Baudizzone, Díaz, Erbin, Lestard, Varas, 1971, p. 20)

Sin embargo, hacia mediados de la década de 1970 las bases fundacionales de la arquitectura de sistemas fueron cuestionadas. Los principales argumentos 
en su contra fueron la dependencia de tecnologías costosas que no lograron ser producidas de manera masiva en el país, el alto consumo energético que demandaba su funcionamiento en un período en que la conciencia ambiental se consolidaba, la excesiva indeterminación funcional que impedía responder con precisión a requerimientos específicos, así como la banalización de la retórica técnica, a medida que las modas posmodernas acentuaban su incidencia (Aliata, 2004). Estas realidades, entre otras, contribuyeron a demostrar que en la arquitectura hay un acotado espacio para la utopía.

\section{REFERENCIAS}

Aliata, F. (2004). Diccionario de arquitectura en Argentina. Tomo S-Z (57-60). Buenos Aires: AGEA.

Aliata, F. (2014). Arqueología de la arquitectura de sistemas. Registros, Revista De Investigación Histórica, 10(11), 6-19. Recuperado de https://revistasfaud. mdp.edu.ar/registros/article/view/49

Altamirano, C. (1998). Desarrollo y desarrollistas. Prismas, Revista de historia intelectual, (2), 75-94. Recuperado de https:/les.scribd.com/document /229296714/Altamirano-desarrollismo-Prismas

Banham, R. (1960). Theory and design in the first machine age. New York: Praeger.

Banham, R. (1969). The architecture of the well-tempered environment. Chicago: University of Chicago Press.

Banham, R. (2001 [1976]). Megaestructuras. Futuro urbano del pasado reciente. Barcelona: Gustavo Gili.

Baudizzone, M.; Díaz, A.; Erbin, J.; Lestard, J.; Traine, E. y Varas, A. (1971). Arquitectarium. Summa, 43 (20).

Baudizzone, M.; Díaz, A.; Erbin, J.; Lestard, J. y Varas, A. (1975a). Tecnología, construir con los medios existentes. Summa, 85(34).

Baudizzone, M.; Díaz, A.; Erbin, J.; Lestard, J. y Varas, A. (1975b). Una aproximación sistemática al diseño. Summa, 85(29).

Baudizzone, M.; Erbin, J.; Lestard, J. y Varas, A. (1980) Architecture. Paris: Presse internationale.

Buchbinder, P. (2005). Una historia de las universidades argentinas. Buenos Aires: Editorial Sudamericana.

Erbin, J.; Varas, A. (1974). Arquitectura para la Universidad. Summa, 83, 18-22.

Gandolfi, F. y Gentile, E. (1997). Ni muros áridos ni calles rectas. El espacio de la Universidad de La Plata, 1897/1975. 47 al Fondo (1), 10-17. Recuperado de http://sedici.unlp.edu.ar/handle/10915/44159

Gentile, E. (2004). Ciudad Universitaria. En J. F. Liernur y F. Aliata. Diccionario de Arquitectura en Argentina. Tomo C-D (83-88). AGEA. 
Malecki, S. (2014). Espacios de mediación: la Ciudad Universitaria de Córdoba, 1949-1962. Registros. Revista De Investigación Histórica (11), 20-39. Recuperado de https://revistasfaud.mdp.edu.ar/registros/article/view/50

Molina y Vedia, J. (23 de agosto de 2004). Entrevista con Miguel Baudizzone. Recuperadadehttp://archivosdarentrevistas.blogspot.com/2012/08/miguelbaudizzone.html

Parera, C. (2017). Pautas para una arquitectura del futuro. Reyner Banham y la tecnología para un entorno bien climatizado. Área, Agenda de Reflexión en Arquitectura, Diseño y Urbanismo (23), 133-145. Recuperado de https:/l area.fadu.uba.ar/area-23/parera23/

Plotkin, M. (2015). La cultura. Argentina (1960-2000). Buenos Aires: Taurus.

Rickert, S. (2006). Recortes en hierro y vidrio. En B. González Montaner (ed.). Del estilo internacional al nuevo futurismo. Patrimonio mundial. Obras y movimientos en los siglos XIX y XX (pp. 72-79). Arte Gráfico Editorial Argentino.

Scott, F. (2007). Architecture or Techno-Utopia: Politics after Modernism. Cambridge: MIT Press.

Silvestri, G. (2014). Alma de arquitecto. Conformación histórica del "habitus" de los proyectistas del hábitat. Registros, Revista de Investigación Histórica, 10(11), 72-97. Recuperado de https://revistasfaud.mdp.edu.ar/registros/ article/view/54/57

Tournikiotis, P. (2001 [1999]). La historiografia de la arquitectura moderna. Madrid: Mairea y Celeste.

Yocelevsky, R. (2015). Una nota sobre el desarrollo de las ciencias sociales en América Latina. Perfiles latinoamericanos, 23(45), 203-222. Recuperado de http://www.redalyc.org/articulo.oa?id=11532721009

Waisman, M. (1995). La arquitectura descentrada. Bogotá: Escala. 